\title{
ALTERNATIVAS DE INTERPRETACIÓN SOBRE LA FORTUNA DE LA PRIMERA BIOGRAFÍA DEDICADA A CARLOS CHÁVEZ
}

Clata Meierovich

\author{
Al maestro Armando Montiel Olvera
}

In Memoriam

No tenemos indicios certeros del año y la ocasión en que Carlos Chávez y Vicente T. Mendoza tuvieron su primer contacto. En este sentido es válido librar conjeturas e imaginar la escenografía del que será, para ambos, fructífero encuentro. Sus vidas transcurrieron casi paralelas en cronologías, contemporizadas por los vínculos efectivos de la pasión artística.

Para esos posibles lazos inaugurales cuenta, sin embargo, el encuentro que sobrevino cuando Chávez es nombrado director del Conservatorio Nacional de Música en diciembre de 1928. ${ }^{1}$ Por su parte, Mendoza finaliza ese año sus estudios regulares en dicho plantel, y meses después ingresa como catedrático para impartir las asignaturas de solfeo y teoría musical. Este momento de conjunción para ambos (comienzos y finales) los lanza encendidos de juventud hacia designios que los erigirán en protagonistas de varias instancias del proceso histórico de la música mexicana.

Entre las múltiples e innovadoras iniciativas emprendidas por el solícito compositor durante su gestión en la jefatura del Conservatorio, que abandonará definitivamente en 1934, se contaba la apertura de la "Clase de creación musical" que instaura a partir de 1931, a la que cambia luego el nombre por el de "Taller de composición", ya que según sus palabras se consideraba un renacentista que creía "más en el taller que en la escuela". Los alumnos participantes en la clase de Chávez eran conducidos gradualmente a la experimentación de una serie de ejercicios melódicos, donde se empleaban escalas diatónicas, cromáticas, pentatónicas e incluso las dodecafónicas, inusuales aún en la música mexicana Las melodías resul-

\footnotetext{
${ }^{1}$ Pocos meses antes el maestro recibió también el encargo de fundar la Orquesta Sinfónica de México, antecesora de la actual Orquesta Sinfónica Nacional.

${ }^{2}$ Roberto García Morillo, Carlos Chávez vida y obra, México, Fondo de Cultura Económica, 1960, p. 62 .
} 
tantes eran interpretadas con instrumentos que se ubicaban en el mismo salón. Dicho procedimiento didáctico ofició de incentivo inmediato a los educandos.

Es probable que el "Taller de composición", iniciado entonces por el maestro, haya sido el primero en ofrecer en México propuestas vitalmente asequibles sobre las potencialidades lúdicas de experimentación y búsquedas musicales, también, claro está, dentro de las alternativas de intuición estética contempladas en los programas de ese momento en el Conservatorio Nacional.

En el "Taller" participaron activamente los talentos en ciernes de Huízar, Revueltas y el propio Mendoza. Posteriormente se unen Ayala, Galindo, Contreras y Moncayo, incorporados a la historia mexicana como el "Grupo de los cuatro", García Morillo incluye también entre los asistentes a Consuelo Cuevas Ney, hoy inescrutable figura del grupo de mujeres compositoras de este país.

No obstante las primeras búsquedas de Mendoza en jurisdicciones de la creación musical, cuando asiste a las clases de composición de Julián Carrillo, ${ }^{3}$ la experiencia de aproximación orgánica con la música obtenida bajo el influjo de Chávez lo determina definitivamente. Tal acercamiento robusteció en él los lazos de admiración y colaboración que, con el transcurrir del tiempo, se tornaron cada vez más recíprocos a la vez que se manifestaron como estímulo irrefrenable para el insospechado Mendozacompositor.

Sin embargo, de todos los alumnos mencionados que se congregaron en torno al maestro Chávez, solamente don Vicente desertará del ferviente cenáculo. Si bien la vocación primera de Mendoza, la música, se abre a través del canal de sus ancestros (abuelo y padre músicos), ésta se irá modulando sin desvirtuar su inicial esencia dirigida a un ámbito científicamente virgen en México: la musicología y la investigación folklorológica.

El ejercicio de don Vicente en el quehacer inherente a la musicología principia con la década de los veinte. ${ }^{4}$ Sin embargo, es desde 1930 cuando comienza con decidido ímpetu y sistemáticamente su colaboración en varias columnas periodísticas capitalinas, y en Armónico séptimo, revista musical de aparición fugaz. Todo este periodismo juvenil atiende una

\footnotetext{
${ }^{3}$ Estudió con Carrillo en varias ocasiones desde 1913 a 1925, asimilando sus enseñanzas de Armonía, Contrapunto, Fuga y prácticas de Composición y Teoría del "Sonido 13". En 1925, convencido de las "maravillas" del sistema de subdivisión del tono inventado por su maestro, Mendoza se convierte, efímeramente, en uno de sus exegetas.

${ }^{4}$ Su trabajo más remoto se fecha justamente en 1920, cuando publica en el Excélsior "Festival de la Orquesta Sinfónica Nacional dedicado a Beethoven", en ocasión en que Carrilo se encontraba al frente de ésta.
} 
temática decididamente musicológica, es decir, una conceptualización teórico-cientifica de la música, así como el rescate y su evaluación histórica. En esta última orientación se ocupó frecuentemente del curso minucioso de los sucesos circunscritos a la música mexicana, extendiendo sus miras hacia el ensayo afiladamente crítico sobre el comportamiento social de la música, reparando tanto en la sensibilidad que emerge del oyente, como en la del artista que se dirige a éste. ${ }^{5}$

En el entreacto de verdadero mare mágnum periodístico que repartía, incluso, entre el ejercicio de la docencia conservatoriana y su devoción siempre latente por el dibujo, el aún incipiente investigador se involucra en un proyecto que logra hasta hoy la indulgencia de la resurrección.

Carlos Chávez y la música mexicana ${ }^{6}$ es, sin lugar a dudas, la biografía más temprana escrita hasta la fecha sobre el polémico compositor y promotor musical. El manuscrito, al que considero indiscutiblemente una revelación, yace desde hace más de veinte años en el "Fondo Vicente T. Mendoza" que custodia actualmente el Fondo Reservado de la Biblioteca Nacional ${ }^{7}$

La fecha en que fue concluido el texto no aparece en el manuscrito. Sin embargo, de acuerdo al período histórico contemplado por Mendoza, podría pensarse que éste llegó a su término al regreso de Chávez de los Estados Unidos, esto es, en julio de 1928. Mi deducción se debe a que el autor escribió en la última página de la obra: "El resto del año 1927 y la mitad del siguiente lo emplea Carlos en estudiar, escribir la reorquestación del Fuego nuevo y en relacionarse ampliamente con multitud de individuos conectados con la música y con todo aquello que con ella se relacione: el cine sonoro y la música eléctrica" (sic).

Queda claro, por otra parte, que la investigación fue gestándose y adquirió reciedumbre al amparo incitante del protagonista. Esta aseveración se encauza sobre la base de que se trata de un itinerario biográfico exhaustivamente minucioso y verídico, no superado, incluso, por el de Garcia Morillo. Difícilmente hubiera podido lograrse, encontrándose el relator a distancia material y emocional de su interpelado.

La razón por la cual el libro finaliza con el segundo viaje del músico al

${ }^{5}$ Dichos intereses quedaron expresados en artículos como "El intérprete musical" (Excélsior, 20 de febrero de 1930), "El auditor musical (Excélsior, 28 de febrero de 1930), "Nuestras academias musicales" (Excélsior, 27 de marzo de 1930) y "El futuro del arte y de los artistas en México" (Excélsior, 7 de agosto de 1930).

${ }^{6}$ Consta de dos legajos: uno de 323 folios y otro más breve de 32 . Las dimensiones de ambos son de $28 \times 21.5$ centímetros, están mecanografiados y no poseen pastas.

${ }^{7}$ La totalidad de la biblioteca, así como gran parte de sus manuscritos fueron vendidos por los hijos de Mendoza a la Universidad Nacional Autónoma de México poco después de la muerte del maestro. 
país vecino, resulta una incógnita difícil de esclarecer. Una explicación cercanamente convincente podríamos hallarla al amparo de que se trató de una primera parte de la biografía, que el investigador se proponía continuar en un futuro estructurando su proyecto en varios tramos temporales, el primero de los cuales consideró pertinente limitar a 1928. Esta fecha es trascendental para el protagonista. Determina el fin de una época de gran receptividad académica y formativa para Chávez, a la vez que suscribe el inicio de una carrera de creciente relevancia pública en la administración oficialista de las artes.

También es válido suponer que la iniciativa del libro partiera del propio compositor, dada la altisonancia de su temperamento, entregando al entusiasta alumno el material que apuntalara con veracidad el estudio, actitud que asumirá en la década de los cincuenta con García Morillo, su biógrafo argentino.

La labor pionera de Vicente T. Mendoza en relación con la difusión de la personalidad y el quehacer de Carlos Chávez, es en la actualidad fácilmente confrontable con el concurso de los documentos que se divulgaron después: estando aún vivo el compositor, como en el caso del libro de García Morillo, y con posterioridad a su muerte, acaecida en 1978, con la aparición de Carlos Chávez, Mexico's Modern-Day Orpheus, de Robert L. Parker, ${ }^{8}$ la tercera biografía, incluyendo al manuscrito de Mendoza.

La constitución temática del libro en su globalidad, y la disposición en partes y capítulos que lo integran, podrían hacer pensar que Mendoza escribió la primera sección de su investigación antes de vislumbrar incluir allí el proyecto biográfico del futuro autor de la partitura Xochipilli-Macuilxóchitl (1940). Pero Mendoza precidió esta sección, o dos primeras partes, como sustancioso basamento histórico a partir del cual comenzó a fundar la biografía. De esta manera queda manifiesto en la breve Introducción que antecede a la obra.

\footnotetext{
Para comprender íntegramente el valor de la personalidad de Carlos Chávez, en vista de todas las características y circunstancias que le rodean, se hace necesario situarlo en el centro preciso de la época en que actúa; pero esto no es bastante; es necesario también mirar retrospectivamente el ambiente musical de México siquiera sea durante el siglo pasado, para no darnos cuenta que en nuestra exigua Historia Musical no existe un antecedente directo a nuestro músico, sino que éste rebasa los límites y su influencia abarca un ámbito inusitado que se remonta varias décadas atrás.

${ }^{8}$ Esta obra fue dada a conocer por la casa editorial Twayne Publishers de Boston, Massachussetts, en 1983
} 
En la metodología empleada por el musicólogo en la obra aludida, se advierte el concurso notorio, y quizá en su manejo inconsciente, de procedimientos positivistas de elucidación histórica. La manera en que el autor se avecina a los hechos que ocupan la realidad comprobable que es la Historia, y en este caso la que concierne al proceso de la música de nuestro país desde el despuntar del siglo XIX, hasta la segunda década del XX, mantiene la distancia de la objetividad y la imparcialidad ideológica; ¿cualidad o defecto que podría caracterizar la conducta académica que mantuvo Vicente T. Mendoza en el curso de toda su labor? A pesar de ello, subyace en las líneas de este trabajo investigador, y en los que luego vendrán de su autoría, el gozo reincidente del escritor y del esteta que logró imprimirle a una gran parte de su obra de creación erudita.

Tampoco la admiración portentosa y perenne por los "folkloristas" Marcelino Dávalos y Rubén M. Campos, ambos considerados bajo las actuales consignas estéticas como estereotipados representantes del decadentismo literario nativista, incidió para que Mendoza transigiera con los lirismos desapegados, o tergiversara la realidad irrebatible de los hechos.

El contenido del Índice total del manuscrito Carlos Chávez y la música mexicana se compendia en cuatro planos, que si bien guardan estrechos vínculos de complementación y concatenación histórica pudieron ser concebidos también como módulos temáticos autónomos entre sí.

\section{Detalle del Índice}

Primera parte: Desarrollo general de la música en México, desde principios del siglo XIX hasta la segunda década del XX.

La música europea importada y su aclimatación en México.

Capitulo I. Usos y costumbres en que intervenía la música.

Capítulo II. Música religiosa.

Capitulo III. Funciones teatrales y demás espectáculos en que intervenía la música.
a. Tonadilla
b. Ballet
c. La ópera
d. La zarzuela
e. Los conciertos

Capitulo IV. Orquestas típicas y bandas militares 
Capitulo $V . \quad$ Enseñanza musical oficial y privada. El Conservatorio.

Capitulo VI. Los músicos y compositores más distinguidos. Principales géneros que se cultivaron.

Segunda parte: Reseña general del desarrollo de las tendencias nacionalistas.

Capitulo I. La música indígena mexicana y su transformación.

Capitulo II. Primeros indicios de atención por parte de la ciudad hacia la llamada música popular.

Capitulo III. Las danzas indígenas y las danzas importadas.

Capitulo IV. Los aires nacionales, primeras recopilaciones y harmonizaciones (sic).

Capitulo $V$. Los aires nacionales, primeras recopilaciones de canciones y corridos.

Capitulo VI. Los pregones y pregoneros.

Capitulo VII. Primeras manifestaciones conscientes de nacionalismo musical.

Capitulo VIII " Los imitadores y continuadores de Manuel M. Ponce.

Capitulo IX. El Departamento de Cultura Estética y sus trabajos nacionalistas.

Tercera parte: Reseña biográfica de Carlos Chávez en relación con el ambiente musical de México.

Capitulo I Garacterísticas del movimiento musical de México hacia 1920 .

Capitulo II. Carlos Chávez. Su presentación en el concierto del 25 de mayo de 1921.

Capítulo III. Primer periodo de producción de Carlos Chávez. Pedro Luis Ogazón. Análisis de las obras de esta época.

Capitulo $V$. Infancia y juventud de Carlos Chávez. Manuel M. Ponce, su influencia sobre Chávez. Las Pehuas de Tlaxcala. Veracruz. "Gladios".

Capitulo V. Características del movimiento cultural 
de México hacia 1921.

Capitulo VI. El fuego nuevo. Principio de una nueva etapa de producción. Influencia de esta obra.

Capitulo VII. Viaje a Europa en 1922-1923. Editoriales contra el academismo. Viaje a Nueva York en 1924. Música nueva. Segundo viaje a Estados Unidos.

Cuarta parte: Apéndice a la primera parte. A la segunda parte.

Luego de revisar cada uno de los cuatro módulos que dan cuerpo al libro, queda la impresión de que las dos primeras partes son el producto de una pertinaz exploración practicada por el musicólogo en hemerotecas y archivos de los teatros de mayor actividad y fuste artístico de la capital y de la provincia. Estas preciadas memorias hoy día han menguado sus acervos hasta alcanzar la extinción.

El texto de la segunda parte del manuscrito también nos da la oportunidad de conocer sus ensayos iniciales en la investigación aplicados a las manifestaciones autóctonas y populares de la música mexicana. Estas incipientes incursiones de Mendoza lo encaminarán, años después, a ahondar y revitalizar cuestiones como el romance español que llegado a estas tierras abrevará (según su tesis) en la esencia formal y lírica del corrido mexicano. Su interés por los "aires nacionales" dará origen a los Aires nacionales del Estado de Hidalgo, estudio escasamente difundido con el cual obtuvo en 1955 el título de "Maestro en Ciencias Musicales" expedido por la Universidad Nacional.

Otro aspecto enigmático en cuanto a la manera en que el autor columbró la obra, y que complica levemente la interpretación del texto, se añade con la presencia del legajo que encontramos adjunto al manuscrito principal, que lleva por encabezado "Desarrollo de la personalidad de Carlos Chávez desde su niñez". Supuestamente, este primer registro biográfico lo escribió para ser inserto al comienzo de la tercera parte, sección central del libro que preludia con la semblanza de su bisabuelo don José Francisco Chávez quien, según el autor, "debió ser criollo o español radicado ya hacía tiempo en México".

El primer biógrafo de Carlos Chávez desarrolla una minuciosa retrospectiva familiar señalando los rasgos más sobresalientes de cada uno de los evocados. Con agilidad de escritura nos revela que José Francisco, asentado en Aguascalientes, transcurrió la mayor parte de su vida en el "Rancho del Alamito" y que "no era ni peón ni siervo, tampoco era terrateniente que 
poseyera grandes superficies de terrenos cultivables; era, sin embargo, un hombre ilustrado que aplicaba métodos apropiados a la labranza de la tierra para hacer a ésta menos ingrata y productiva” "De su bisabuela, doña Victoriana Alonso, sugiere un perfecto retrato decimonónico; estaba dotada de "grandes prendas morales, hogareña y dócil, como todas las de la época, probablemente hija de españoles radicados en el país, como nos lo hace sospechar el apellido Alonso, propio de las familias criollas de la región". Del matrimonio nacieron José María, Ignacio y Julián. El primero, abuelo del compositor, quien fungió como destacada figura política de su estado (fue gobernador interino) y activo defensor contra el invasor yankee.

Entre los seis o siete años el artista biografiado presiente su inclinación por la música, cuando asiste con su madre a los conciertos sinfónicos dirigidos por Carlos J. Meneses, ${ }^{9}$ "quien desde entonces adiestraba sus oídos a conjuntos de orquesta".

Mendoza describe los pasos subsecuentes de Chávez con sus primeros maestros: Asunción Parra, Ponce y Ogazón, hasta llegar a Juan B. Fuentes: el influjo musical más determinante en su juventud. El legajo finaliza con el nombramiento que se le hace como Inspector de Música del Departamento de Cultura Estética, por parte del subsecretario doctor Gastélum: "Ocupando este puesto trabajó en un Centro Nocturno de Obreros en donde principió a enseñar los cantos indígenas de la Sierra Andina, Colección D'Harcourt que trajera de su viaje a Europa; ${ }^{10}$ pero mirando el Jefe Sr. Beristáin los resultados obtenidos intrigó de tal modo que obligó al joven Inspector a presentar su dimisión." Según testimonia Mendoza, ello ocurrió cuando el músico contaba solamente veinticuatro años.

También cabe la posibilidad de que el destino de esta breve sección fuera otro proyecto con diferentes ambiciones o intenciones por parte del investigador. Pudo ser concebido con anterioridad al legajo principal, con la finalidad de darlo a conocer en forma de artículo en alguna revista o publicación especializada de la época.

Resulta un hecho innegable que Mendoza tuvo buen tino en la elección de su personaje. Presintió la relevancia que llegaría a alcanzar Chávez en la iniciativa y conducción de la política musical y artística del país, y vislumbró la consecuencia estética que traería aparejada la misión de su obra. En este

\footnotetext{
${ }^{9} \mathrm{Al}$ maestro Meneses (1863-1929) se le considera el primer director de orquesta mexicano. Dirigió largas y novedosas temporadas de repertorio con la primigenia Orquesta Sinfónica, que se fundó con el patrocinio del Estado. Ofreció con esta agrupación una actuación ininterrumpida desde 1902 a 1912.

${ }^{10}$ Este viaje lo realizó en compañía de su esposa Otilia Ortiz. Visitaron Viena, Berlín y París entre septiembre de 1922 y abril de 1923.
} 
sentido, Carlos Chávez fue un auténtico derribador de modalidades obsoletas: un "agitador" como lo señaló José Gorostiza en alguno de sus ensayos críticos . Removió los procedimientos composicionales que hasta el promediar de los treinta, aproximadamente, permanecían como los únicos y socorridos rudimentos de que hacían acopio nuestros músicos, algunos de ellos empecinados aún en la libación de las técnicas acartonadas que habían adquirido en las aulas europeas, pero que en aquella hora comenzaban a desencajar del flujo de cambios evolutivos que aportaban los nuevos sistemas musicales, como por ejemplo el dodecafonismo y el serialismo, amén de la utilización cada vez más frecuente de la politonalidad y la polirritmia exhibidas en las partituras de compositor es europeos y estadounidenses. Frente al podio de la Orquesta Sinfónica de México, Chávez comenzó a desvanecer la inmovilidad que encarnaba en las programaciones, ofreciendo a los públicos ávidos de escuchar renovados repertorios sinfónicos, obras en las que se asumían las nuevas posturas estéticas de entonces.

Tampoco este trabajo fue el único ni el último que dedicó el musicólogo a la promoción del celebrado artista. En el mismo año en que aparece su hasta hoy encomiable a la vez que controvertido estudio El romance español y el corrido mexicano (1939), da a conocer un breve texto que titula "Técnica de Carlos Chávez compositor" " 11 En tono de veneración sin límites por el músico-creador, señalaba allí:

Carlos Chávez es un músico de cinco sentidos actuando en la producción conjuntamente; su desarrollo cerebral le permite percibir íntegramente, en el momento de la gestación musical de sus obras, hasta los últimos detalles; produce simultáneamente melodía, armonía y ritmo, dentro de las condiciones enunciadas, es decir, melodía en forma polifónica, armonía politonal, polirritmia, timbre instrumental utilizado como doble contrapunto, todo esto encuadrado dentro de formas universalmente aceptadas. Por todas estas causas, y por la manera de utilizar estos elementos técnicos, Carlos Chávez es actualmente un músico integral, moderno, en potencia activa, en contacto directo con la vida y con el elemento sonoro que cultiva...12

Años más tarde Mendoza extracta y afina algunos de los juicios vertidos en la biografía primigenia, en un artículo que bajo el escueto encabezado de "Bosquejo biográfico" presenta a los lectores del periódico El Nacional el 27 de julio de 1955. Este compendio marca su última aproximación, por lo

${ }^{11}$ En Anales del Instituto de Investigaciones Estéticas, tomo II, núm. 3, año III, 1939.

${ }^{12}$ Op. cit., p. 24. 
menos la última hasta la fecha conocida, sobre el suculento "caso Chávez".

El afán de Vicente T. Mendoza por disipar las sombras que acechaban la verdad del pasado musical de las primeras décadas, logró convincentes alcances en las páginas pioneras del manuscrito Carlos Chávez y la música mexicana, obra de preliminares para estudios que llegarán después a completar y actualizar el retrato del aureolado artista. Es de lamentar, por otra parte, que Roberto García Morillo, sucesor de Mendoza en la tarea de reanudación biográfica, haya sido escueto en el reconocimiento de los créditos al investigador mexicano. En su libro, que ya hemos citado páginas atrás, alude elogiosamente en varias ocasiones al esfuerzo indagador dispensado por Mendoza al quehacer del maestro Chávez. En la síntesis bibliográfica que apoya su investigación, menciona únicamente como fuente de sus acotaciones documentales, el texto de "Bosquejo biográfico" al que ya nos hemos remitido. Este hecho resulta evidentemente falso. Ello se deriva de la confrontación del texto de las citas pertenecientes a Mendoza, con el propio artículo periodístico, en el cual no aparecen tales revelaciones u opiniones. Esta actitud escasamente honesta por parte del compositor y crítico argentino deja implícita la sospecha de que Morillo tuvo alguna vez en sus manos las páginas de este manuscrito, sin que localicemos pistas de la manera o los canales por los que éste llegó a él. Respecto a esta situación, no debemos excluir la posibilidad de que Chávez, conocedor seguramente del texto, haya conservado una copia del libro que prestó a Morillo en vísperas de la redacción de su obra.

Con la sinopsis histórica oficiando como certero encuadre de la gestión artística y administrativa de Carlos Chávez, el maestro Mendoza dio los primeros pasos firmes en el ámbito disciplinario de la investigación musicológica, analizando el comportamiento de la música europea y su resultado sincrético en la configuración de la música mexicana.

Ante esta peculiar obra surge naturalmente la pregunta con respecto al silencio de casi seis décadas en que permaneció sumido el manuscrito. El recurso factible de la lógica, y el auxilio a veces inexorable de la intuición, inducen a considerar que el autor sintió entonces su libro como poco "sazonado", resguardándolo de las críticas recelosas de sus colegas, con miras, quizás, a reforzarlo en algún momento de su tiempo futuro.

El inédito al cual nos referimos configura un muy apreciado instrumento historiográfico que, en conjunción con la Historia de la música en México (1934) de Gabriel Saldívar, ofrece una satisfactoria apreciación retrospectiva de la circunstancia musical de nuestro país en épocas pretéritas, sobre las que aún nos queda mucho por aclarar. 